\title{
Indispensability and supremacy of clinical examination \& monitoring over modern instrumental examination \& monitoring in anesthesia
}

\section{Introduction}

There are verses in the Holy Quran said:

i. "Man is hasty by nature!"

ii. "Oh believer! Seek help with patience \& prayer; surely, Al lah is with the steadfast". ${ }^{2}$

"Vigilance" is the motto and slogan of the American Society of Anesthesiologists (ASA). Conduct of anesthesia is extremely hazardous \& complex monitoring work, per se, demands constant vigilance. Deplorable fact is, by nature human being are not dependable $\&$ patience at monitoring because they are error prone and vigilance work is prone to deterioration by various human, environmental, and equipment factors. ${ }^{3}$

\section{Significance \& necessity of clinical examination \& monitoring}

In spite of advancement in technology, clinical examination \& monitoring plays a significant role as the anesthesia machines $\&$ equipments might collapse at any stage. Monitor \& machine derived parameters need to be confirmed by clinical means which comprises extensive monitoring of anesthetized patients, anesthesia machine \& equipment and events inside operating theatre. Depth of anesthesia may be determined by clinical parameters for instance pulse, respiration/movements, systolic blood pressure, tear (PRST). Bed side cardiovascular parameters may be monitored by palpating peripheral pulses, seeing capillary refill and auscultating heart sounds. Similarly respiratory parameters may be monitored by observing chest movements, watching \& feeling reservoir bag, auscultating lung fields. Again cyanosis \& anemia can be observed by colour of lips and nail beds as well. ${ }^{4}$

AAGBI has prepared \& published guidelines for minimum standards of monitoring during anesthesia. An expert anesthesiologist should be present throughout the period of anesthesia. Anesthesiologist should screen \& televise clinical parameters of monitoring equipments frequently and should undertake appropriate timely interventions to fix \& optimize those. Anesthetic equipments should be checked \& calibrated before use as per the manufacturer's instructions. All alarms should be enabled during perioperative period and alarm limits must be set at suitable values for individual patients based on age $\&$ sex. ${ }^{4}$

\section{Evolution \& limitations of instrumental monitoring}

During anesthesia, to ensure optimum patient safety certain core standards of monitoring is followed, which provides very useful \& valuable information's eventually facilitates early detection \& diagnosis as well as management of critical incidents. Experienced and vigilant anesthesiologist is the most important monitor who is the only alive $\&$ passionate person between sleeping patient $\&$ lifeless equipments. Though it is quiet challenging to prevent each \& every

\author{
Volume 4 Issue 3 - 2016 \\ Lt Colonel Abul Kalam Azad \\ Associate Prof Anesthesiology \& Intensive Care, Combined \\ Military Hospital, Bangladesh \\ Correspondence: Lt Colonel Abul Kalam Azad, Classified \\ Specialist/Associate Prof Anesthesiology \& Intensive Care, \\ Combined Military Hospital, Post Code- I206, Dhaka \\ Cantonment, Dhaka, Bangladesh, Tel 008801715010956, \\ Email azad100864@gmail.com
}

Received: January 21, 2016 | Published: February 12, 2016

adverse events, as human error is inevitable. Constant monitoring gives an early warning eventually reduces the consequences of critical incidents. Hence, manufacturers of anesthetic equipment have strived to "aid" the anesthesiologist by incorporating sensors, devices and systems to enhance vigilance and clinical performance. Anesthesia machine \& equipments need monitoring along with patients and eventually derived parameters demand validation as well. ${ }^{4}$

\section{Conclusion}

To maintain normal patient's physiology \& homeostasis and to ensure adequacy of anesthesia need continual assessment and monitoring. Monitoring devices assist anesthesiologist to ensure integrity of vital organs. Devices can only supplement not substitute clinical observation \& judgement in order to achieve target of monitoring. Despite sophisticated anesthesia machines \& equipments the presence of a properly trained competent anesthesiologist is the prime determinant of patient safety during anesthesia. Anesthesiologists must be aware of their limitations and hazards, including human failure and ensure best $\&$ judicious use of these machines. Monitoring can't anticipate \& stop all adverse events or accidents throughout peri-operative period. Certainly, there is substantial evidence that it reduces the risks of adverse events and accidents both by early detection of the consequences of errors, and by early warning that the clinical condition of a patient is deteriorating.

\section{Acknowledgments}

None.

\section{Conflicts of Interset}

None.

\section{References}

1. Surah Bani Isra'il The Holy Quran. Verse 12 Chapter 17. 
2. Surah Al-Baqarah The Holy Quran. Verse 154 Chapter 2.

3. Matthew BW, Ty Smith N. Vigilance, Alarms, and Integrated Monitoring Systems. Chapter 18 1994. p. 4-7.
4. Mendonca C. Monitoring During Anaesthesia and Recovery. Warwick Medical School- Handbook of Anaesthesia. 2006. p. 1-11. 